\title{
Case Studies and the Statistical Worldview: Erratum
}

\author{
Timothy J. McKeown
}

McKeown's review article in the Winter 1999 International Organization (volume 53 , no. 1, pages 161-90) contains an error in footnote 65 on page 179 . The correct text should read:

65. The Social Sciences Citation Index shows that Cohen and Axelrod's paper has been cited only fourteen times in the last ten years. 